01

\title{
Метод трансляционного переноса для оценки стабильности нестационарного квантового стандарта частоты
}

\author{
(C) С.В. Божокин, К.А. Баранцев, А.Н. Литвинов \\ Санкт-Петербургский политехнический университет Петра Великого, \\ 195251 Санкт-Петербург, Россия \\ e-mail: bsvjob@mail.ru
}

Поступило в Редакцию 30 апреля 2020 г.

В окончательной редакции 22 июня 2020 г.

Принято к публикации 22 июня 2020 г.

Непрерывное вейвлет-преобразование применено для анализа работы нестационарного сигнала квантового стандарта частоты. Предложен метод трансляционного переноса, с помощью которого устранены граничные явления в этом преобразовании. Вычислены спектральные интегралы сигнала квантового стандарта частоты в различных диапазонах частот. Введена вейвлет-дисперсия, позволяющая определить моменты времени, когда флуктуации сигнала наиболее сильны. Проведено сравнение вейвлет-дисперсии с обычной дисперсией и с дисперсией Аллена.

Ключевые слова: непрерывное вейвлетное преобразование, квантовый стандарт частоты, вейвлетдисперсия, нестационарный сигнал.

DOI: $10.21883 /$ JTF.2021.01.50269.152-20

\section{Введение}

Известно, что спутниковые навигационные системы имеют своей целью создание глобального координатновременного поля, доступного в любой точке Земли и в космосе, для высокоточного измерения координат, навигации, управления, синхронизации приема-передачи информации между удаленными объектами. Ключевыми элементами, определяющими точность синхронизации всех бортовых и наземных элементов спутниковых систем навигации, являются квантовые стандарты частоты (атомные часы) [1-5]. Они составляют основу навигационных систем ГЛОНАСC, GPS, а также разрабатываемой в Европе системы GALILEO, в Китае системы Бэйдоу, и в Индии системы IRNSS. Замена существующих стандартов частоты бортового базирования, связанная как с естественным выходом из эксплуатации работающих устройств, так и с проектированием новых систем, предполагает использование более совершенных в техническом отношении решений.

Одной из главных характеристик квантового стандарта частоты (QFS) является его кратковременная и долговременная стабильность. Несовершенство квантового дискриминатора (тепловые, дробовые и фликкер шумы) определяют кратковременную стабильность QFS. Различные температурные эффекты, деградация источников и приемников оптического излучения определяют долговременную стабильность QFS. На стабильность работы QFS также влияют флуктуации сигнала оптического детектирования, нестабильность параметров электромагнитного излучения, неидеальность фотодетекторов, нестабильность источников питания, неоднородности температурного поля идр. Для определения как кратковременной, так и долговременной стабильно- сти традиционно используется критерий, основанный на вычислении дисперсии Аллена $[4,6,7]$. Формула для дисперсии Аллена выведена в предположении нормального, стационарного, эргодического случайного процесса, однако в реальных условиях и при длительной работе прибора мы имеем дело с многочисленными переходными процессами, следствием которых является нестационарность шумов сигнала дискриминатора. Это означает, что статистические и спектральные свойства, вычисленные для достаточно большого интервала времени, изменяются. Другими словами, если взять интервал времени, смещенный на произвольную величину относительно первоначального интервала, и сопоставить с исходным интервалом, то статистические и спектральные свойства сигналов на этих интервалах не будут совпадать. Разумно предположить, что в течение работы QFS нестационарные шумы с изменяющими спектральными характеристиками могут возникать в какие-то моменты времени, и также исчезать в последующие моменты времени.

Несмотря на то, что в подавляющем большинстве работ, посвященных анализу стабильности (QFS), расчеты выполнены в рамках аллановского подхода [8$10]$, имеется ряд работ по изучению алгоритмов обработки сигнала, учитывающих нестационарность шумов сигнала. Основу этих алгоритмов составляет вейвлетанализ [11-16]. Основная новизна подхода состоит в том, что при вычислении непрерывного вейвлетного преобразования используется адаптивный вейвлет Морле, позволяющий изменять временное и частотное разрешения исследуемых сигналов с помощью вспомогательного управляющего параметра [17-19]. В [20] метод непрерывного вейвлетного преобразования применен для анализа нестационарного дискретного сиг- 
нала квантового стандарта частоты (QFS). Однако данная работа имеет определенный недостаток, а именно, при анализе сигнала QFS не учитываются граничные условия, возникающие при реализации непрерывного вейвлет-преобразования. Это обстоятельство позволило сформировать тематику настоящей работы.

Таким образом, целью настоящей работы является развитие теории вейвлетов для анализа нестационарных сигналов QFS с учетом граничных условий. При этом непрерывное вейвлетное преобразование (CWT continuous wavelet transform) будет обобщено на случай дискретного сигнала. Спектральные интегралы, характеризующие работу QFS в различные моменты времени, будут вычислены для всех диапазонов частот. В заключительной части работы проведено сравнение вейвлет-дисперсии (дисперсия, характеризующая нестационарную работу QFS) с традиционными методами, оценивающими стабильность работы QFS.

\section{1. Анализ стабильности QFS с помощью дисперсии Аллена}

Рассмотрим анализ стабильности QFS с помощью дисперсии Аллана [6]. Определим относительную частоту сигнала QFS, как $y(t)=\left(v(t)-v_{0}\right) / v_{0}$, где $v(t)-$ измеренная частота в момент времени $t$, которая отличается от эталонного значения $v_{0}$ вследствие различных флуктуаций. Предположим, что QFS paботает в течение времени $T$. Определим $T=N \Delta t$, где $N-$ число точек, а $\Delta t-$ время дискретизации. В этом случае сигнал $y_{n}=y\left(t_{n}\right)$ становится дискретным, причем моменты времени $t_{n}=n \Delta t$, где $n=0,1, \ldots N-1$. Спектр частот различных флуктуаций с частотой $f$ также становится дискретным $f_{k}=k / T$, где $k=1,2 \ldots$ - целые числа. Рассмотрим модельный сигнал QFS $(N=131072, \Delta t=0.5 \mathrm{~s})$, для которого вычислим среднее значение $\langle y\rangle=\frac{1}{N} \sum_{n=0}^{N-1} y_{n}$, и выборочную дисперсию $\sigma^{2}=\frac{1}{N} \sum_{n=0}^{N-1}\left[y_{n}-\langle y\rangle\right]^{2}$. На рис. 1 построена функция $\sqrt{y^{2}(t)} / \sigma$ в зависимости от времени $t$. Для данного QRS величина кратковременной стабильности $\sigma=2.3 \cdot 10^{-12}$. Из этого графика можно видеть, что сигнал $y(t)$ имеет сильные флуктуации, причем в максимуме величина $\sqrt{y_{\max }^{2}(t)} / \sigma \approx 4.5$.

Построим спектр мощности сигнала $P\left(f_{k}\right)=|\hat{y}(k)|^{2}$ (рис. 2), который может быть выражен через фурьегармоники $\hat{y}(k)$ этого сигнала $y(t)$. Спектр мощности $P\left(f_{k}\right)$, нормированный на единицу, спадает в зависимости от частоты $0<f_{k}<0.75 \mathrm{~Hz}$. Двухвыборочная дисперсия Аллена $\sigma_{A l}^{2}(\tau)$, зависящая от текущего времени $\tau$, в настоящее время является наиболее распространенной мерой стабильности частоты QFS [8-10]. Для дискретного набора частот $f_{K}=k / T$ формула для

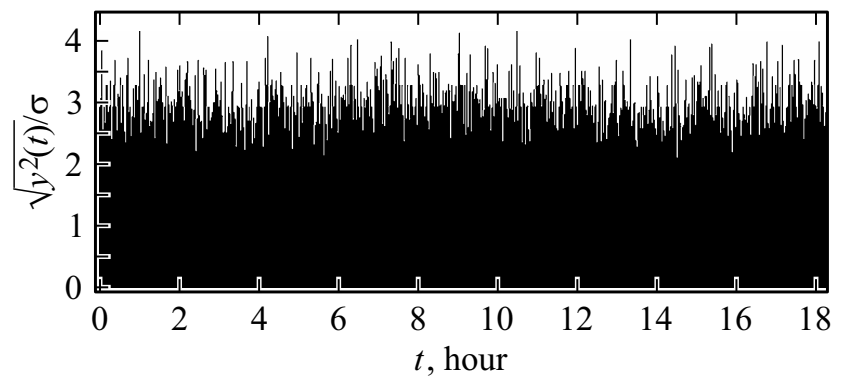

Рис. 1. Зависимость $\sqrt{y^{2}(t)} / \sigma$ от времени $t$ для $Q R S$.

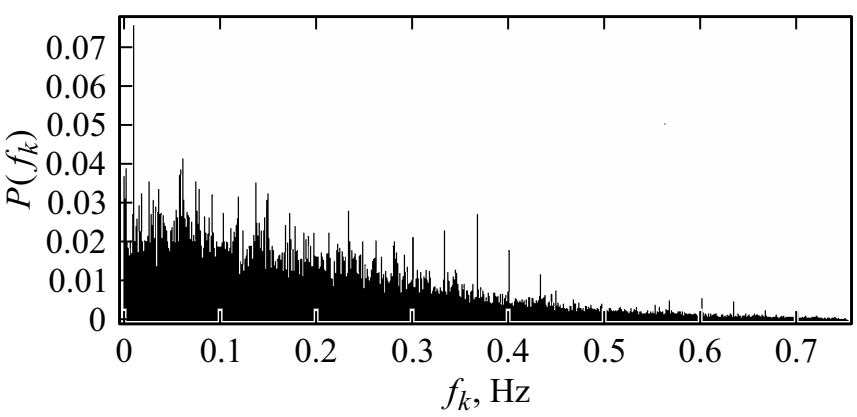

Рис. 2. Спектр мощности $P\left(f_{k}\right)$ сигнала $y(t)$, нормированный на единицу, в зависимости от частоты $f_{k}$.

дисперсии Аллена имеет вид:

$$
\sigma_{A l}^{2}(\tau)=4 \sum_{k=0}^{N / 2} \frac{\sin ^{4}(\pi k \tau / T)}{(\pi k \tau / T)^{2}}|\hat{y}(k)|^{2} .
$$

Известно, что формула дисперсии Аллена $\sigma_{A l}^{2}(\tau)$ опирается на измерение разности средних для двух соседних последовательных измерений частоты. Это определение отличается от определения дисперсии, когда наблюдаемые величины сравниваются со средним значением по всему интервалу измерений $[0 ; T]$. В основе вывода $\sigma_{A l}^{2}(\tau)$ лежит условие эргодичности стационарного процесса. Следовательно, усреднение по статистическому ансамблю эквивалентно усреднению по времени. Однако в реальной ситуации предположение о стационарности работы QFS может нарушаться. Это связано с тем, что в работе реального устройства имеются флуктуации сигнала оптического детектирования, нестабильность параметров электромагнитного излучения, неидеальность фотодетекторов, нестабильность источников питания, неоднородности температурного поля и др.

Вычислим $\sigma_{A l}(\tau)$ для данного QFS. Подставляя явный вид спектра мощности в (1) (рис. 3) и выполняя суммирование, получаем, что величина $\sigma_{A l}(T / 2)=1.99 \cdot 10^{-14}$. Недостатком формулы дисперсии Аллена является то обстоятельство, что спектр мощности сигнала $P\left(f_{k}\right)=|\hat{y}(k)|^{2}$ может только указать на присутствие определенных спектральных гармоник. Различная динамика возникновения и исчезновения 


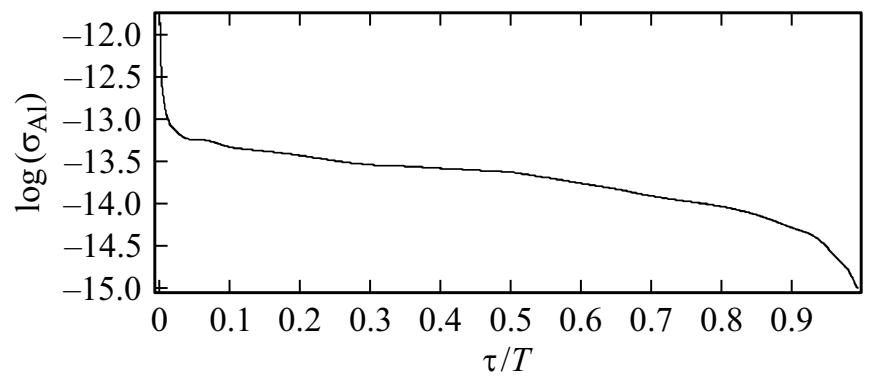

Рис. 3. Зависимость десятичного логарифма $\log \left(\sigma_{A l}(\tau)\right)$ от безразмерного времени $\tau / T$, где $T-$ период наблюдения.

вспышек активности, возникающая в различные моменты времени в различных спектральных диапазонах, не отражена в формуле (1). Это требует разработки нового подхода, описывающего нестабильность работы QFS, учитывающего нестационарность сигнала $y(t)$. Этот новый подход будет представлен ниже.

\section{2. Непрерывное вейвлет-преобразование сигнала QFS}

Для анализа нестационарных сигналов в теории вейвлетов широко используется непрерывное вейвлетпреобразование (Continuous Wavelet Transform) [11-16]. Оно имеет преимущество перед оконным преобразованием Фурье (STFT - Short Time Fourier Transform), поскольку STFT использует постоянное окно продолжительностью $W$, в то время как CWT использует адаптивное окно. Преимущество использования адаптивного окна в CWT состоит в том, что на малых частотах окно становится широким, а на высоких частотах узким. Это преимущество CWT перед STFT, так как явный вид STFT зависит от размера окна $W$. Для многих нестационарных сигналов, имеющих различные продолжительности вспышек с разными частотами, оптимальный выбор окна является сложной задачей.

Обозначим $V(v, t)$ непрерывное вейвлетпреобразование, зависящее от частоты $v$ и времени $t$. Вместо обычной частоты $v$, имеющей размерность $\mathrm{Hz}$, введем безразмерную частоту $Q=v \Delta t$. Момент времени $t$ переходит в дискретную величину $t_{n}=n \Delta t$, где $n=0,1, \ldots N-1$. Тогда непрерывное вейвлетпреобразование $V(Q, n)$ дискретного сигнала $y(n)$ будет иметь вид

$$
V(Q ; n)=Q \sum_{n^{\prime}=0}^{N-1} y\left(n^{\prime}\right) \Psi^{*}\left[Q\left(n^{\prime}-n\right)\right] .
$$

Здесь величина $V(Q, n)$ отображает одномерный сигнал $y(n)$ на плоскость изменяющихся аргументов $\{Q ; n\}$ : безразмерной частоты $Q$ и времени $n$. Комплексная функция $\Psi(x)$ представляет собой материнский вейвлет, пространственная протяженность которого равна $\Delta_{x}$. Выражения для материнского вейвлета $\Psi(x)$, а также его Фурье компоненты $\hat{\Psi}(F)$ приведены в работах [17-19]. $\mathrm{C}$ помощью непрерывного вейвлетного преобразования можно ввести среднее значение локальной плотности спектра энергии сигнала $\varepsilon(Q ; n)$ :

$$
\varepsilon(Q ; n)=\frac{1}{C_{\Psi}} \frac{|V(Q ; n)|^{2}}{Q},
$$

где

$$
C_{\Psi}=\int_{0}^{\infty} \frac{|\hat{\Psi}(F)|^{2} d F}{F}
$$

Спектральный интеграл $E_{\mu}(n)$, введенный в [17-19]:

$$
E_{\mu}(n)=\frac{1}{\Delta Q} \int_{Q_{\mu}-\Delta Q / 2}^{Q_{\mu}+\Delta Q / 2} \varepsilon(Q, n) d Q
$$

представляет собой среднее значение локальной плотности спектра энергии сигнала $\varepsilon(Q ; n)$, проинтегрированное по определенному диапазону частот

$$
\mu=\left[Q_{\mu}-\Delta Q / 2 ; Q_{\mu}+\Delta Q / 2\right]
$$

где $Q_{\mu}$ - середина частотного интервала, $\Delta Q-$ размер интервала частот. Изучая поведение спектрального интеграла $E_{\mu}(n)$, можно анализировать явления появления и исчезновения вспышек спектральной активности сигнала в диапазоне частот $\mu$ в зависимости от дискретного времени $t_{n}=n \Delta t$.

Введем вейвлетную дисперию $\sigma_{W}^{2}(n)$, представляющую собой интеграл от спектральной плотности, вычисленный по всему спектральному диапазону

$$
\sigma_{W}^{2}(n)=\int_{0}^{\infty} \varepsilon(Q, n) d Q .
$$

Для вейвлетного преобразования дискретного сигнала справедлив аналог равенства Парсеваля

$$
\left\langle y^{2}(n)\right\rangle=\frac{1}{N} \sum_{n=0}^{N-1} y_{n}^{2}=\frac{1}{N} \sum_{n=0}^{N-1} \sigma_{W}^{2}(n) .
$$

Вейвлетная дисперсия $\sigma_{W}^{2}(n)$, зависящая от конкретного интервала времени $t_{n}=n \Delta t$, представляет собой величину, усредненную как по временам с помощью адаптивного окна, зависящего от частоты $Q=v \Delta t$, так и по всем частотам $Q$. Отметим, что при выборе окна вейвлетного преобразования существенным становится проблема, связанная с граничными условиями на краях этого окна. Следующий раздел будет посвящен этому вопросу. 


\section{3. Устранение граничных условий при выполнении CWT}

Особенностью численных методов вычисления $\mathrm{CWT}$ (2) для различных времен $t \rightarrow t_{n}=n \Delta t$ и частот $v \rightarrow \frac{Q}{\Delta t}$ является появление граничных эффектов, проявляющихся как на левой $\left(0<t<t_{o f f}\right)$, так и на правой границе $\left(T-t_{o f f}<t<T\right)$ промежутка наблюдения сигнала $T$. Для их устранения обычно результаты $V(Q, n)(2)$, получающиеся как для $0<t<t_{o f f}$, так и в правом граничном интервале $T-t_{\text {off }}<t<T$, обнуляются. Естественно, что в этом случае теряется ценная информация о частотно-временном поведении сигнала $y(t)$ в этих граничных интервалах. Поясним это более подробно. Для этого введем параметр локализации $\Delta_{x}$, определяющий протяженность материнского вейвлета $\psi(x)$ по оси $x$. Минимальная частота исследуемого сигнала $v_{\min }$ связана с моментом времени $t_{o f f}$ соотношением $t_{o f f}=\frac{2 \Delta_{x}}{v_{\min }}$. Аналогичный промежуток времени $\left[T-t_{o f f} ; T\right]$ будет и на правом участке интервала времени $[0 ; T]$. Для определения частоты $v_{\min }$ необходимо, чтобы на оставшемся интервале времени, равном $T-2 t_{o f f}$, помещалось $K$ периодов колебаний частоты, равных $1 / v_{\min }$, причем величина $K \gg 1$, поэтому $v_{\min }=\left(K+4 \Delta_{x}\right) / T$. Отсюда становится ясно, что учет граничных эффектов приводит к тому, что величина $v_{\min }$ примерно в $K+4 \Delta_{x}$ раз больше, чем минимальная частота $f_{\min }=1 / T$, используемая в фурье-анализе.

Для устранения граничных эффектов нами был предложен следующий алгоритм, основанный на трансляционном переносе сигнала (TST - translation signal transfer):

1) по известной величине $v_{\min }$ можно расширить интервал изучения сигнала $y(t)$, увеличив его с величины $T$ до величины $T_{1}$;

2) начало интервала $T(t=0)$ переносится в точку $t_{\min }$. Весь начальный промежуток $\left[0 ; t_{\min }\right]$ нового интервала наблюдения сигнала $T_{1}$ заполняется постоянными значениями сигнала в начальный момент времени $y(t=0)$. При этом в качестве величины $t_{\min }$ выбирается величина, большая, чем начальный участок, где будут наблюдаться граничные эффекты CWT $t_{\min }=2.5 \Delta_{x} / v_{\min }$. Отметим, что величина $t_{\min }$ немного превосходит величину $t_{o f f}$, так как $t_{\min }=1.25 t_{\text {off }}$;

3) конечный промежуток нового интервала $\left[T_{1}-t_{\min } ; T_{1}\right]$ также заполняется постоянными значениями сигнала в конечный момент времени наблюдения $Z(t=T)$. В этом случае центральный интервал $\left[t_{\min } ; T_{1}-t_{\min }\right] \quad$ промежутка $T_{1}>T, \quad$ имеющий продолжительность $T$, в точности повторяет все значения исследуемого сигнала $y(t)$, заданного на этом же промежутке наблюдения;

4) приравняем значения минимальной частоты $v_{\min }(T)=-v_{\min }\left(T_{1}\right) \quad$ для периода $T \quad$ и периода $T_{1}=T+5 \Delta_{x} / v_{\min }$. В этом случае число периодов $K(T)$ и $K_{1}\left(T_{1}\right)$ связаны соотношением $K_{1}\left(T_{1}\right)=K(T)+5 \Delta_{x}$.

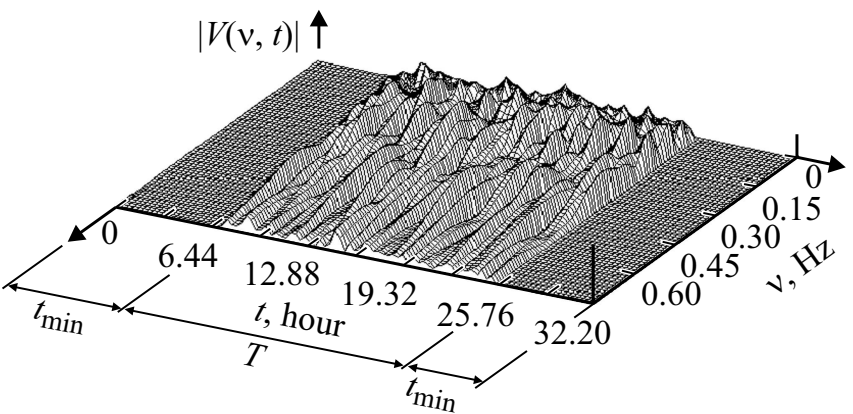

Рис. 4. Зависимость модуля CWT $|V(v, t)|$ от частоты $v$ и времени $t$.

Рассмотрим численную реализацию CWT на интервале времени $\left[0 ; T_{1}\right]$. На рис. 4 показан CWT-сигнал $V(v, t)$, который сдвинут с помощью метода TST. Начальный $\left[0, t_{\min }\right]$ и конечный интервал $\left[T_{1}-t_{\min } ; T_{1}\right]$ расширенного интервала $\left[0, T_{1}\right]$ заполняются постоянными граничными значениями сигнала, которые вносят нулевой вклад в $V(v, t)$. Таким образом, граничные эффекты в первоначальном интервале сигнала $[0, T]$ оказываются учтенными. Проанализируем численные данные сигнала на рис. 4. Для минимальной частоты $v_{\min } \approx 2 \cdot 10^{-4} \mathrm{~Hz}$ период наблюдения за сигналом длительностью $T=18.2 \mathrm{~h}$ увеличивается до величины $T_{1}=32.2 \mathrm{~h} \quad\left(T_{1}=T+2 t_{\min }\right)$. Ранее граничные эффекты исследуемого сигнала $y(t)$ проявляли себя в диапазоне низких частот $v \geq v_{\min }$ на интервалах времени $0<t<t_{\text {off }}$ и $T-t_{\text {off }}<1 \leq T$. Примененный метод TST приводит к тому, что граничные эффекты при расширении интервала наблюдения сигнала $0 \leq t \leq T_{1}$ оказываются в центральной части нового интервала $T_{1}$, вне области действия граничных эффектов CWT.

На втором этапе применения метода TST отбрасываются значения $V(v, t)$, расположенные в начале интервала $\left[0 ; t_{\min }\right]$ и в конце интервала $\left[t_{\min } ; T_{1}-t_{\min }\right]$. Последующий перенос начала отсчета времени из точки $t=t_{\min }$ в точку $t=0$ восстанавливает первоначальный интервал наблюдения $t=[0 ; T]$ и позволяет сравнивать значения $V(v, t)$ с учетом граничных условий с истинным поведением сигнала $y(t)$. Такой обратный перенос сигнала восстанавливает первоначальную шкалу времени $[0, T]$. Одновременно это позволяет правильно учесть низкочастотные флуктуации сигнала, которые происходят на левом и на правом краю этого интервала $[0, T]$.

Таким образом, метод TST, примененный для численной реализации CWT, позволил сохранить важную информацию об амплитудно-частотном поведении сигналов вблизи начала и вблизи конца интервала наблюдения. 


\section{4. Вычисление спектральных интегралов $E_{\mu}(t)$}

Во Введении было сказано о том, что метод оценки стабильности, основанный на анализе дисперсии Аллена $\sigma_{A l}(\tau)$, не может обнаружить моменты времени $t$, в которых происходят сильные флуктуации сигнала QFS в различных спектральных диапазонах. Для того чтобы решить эту задачу, разработаем технику спектральных интегралов, основанных на CWT. Для этого разобьем весь диапазон частот $v$ на некоторые спектральные диапазоны $\mu=\{U L F, V L F, L F, H F\}$, имеющие следующие границы:

$$
\begin{gathered}
U L F=\left[v_{\min } ; F_{U L F}\right], \quad V L F=\left[F_{U L F} ; F_{V L F}\right], \\
L F=\left[F_{V L F} ; F_{L F}\right], H F=\left[F_{L F} ; F_{H F}\right],
\end{gathered}
$$

причем $\quad v_{\min }=1.984 \cdot 10^{-4} \mathrm{~Hz}, \quad F_{U L F}=0.030 \mathrm{~Hz}$, $F_{V L F}=0.08 \mathrm{~Hz}, \quad F_{L F}=0.3 \mathrm{~Hz}, \quad F_{H F}=0.75 \mathrm{~Hz}$. Такое деление на поддиапазоны $\mu$ соответствует делению всего спектра на сверхнизкие ULF (ultra low frequencies), очень низкие VLF (vary low frequencies), низкие LF (low frequencies) и высокие $\mathrm{HF}$ (high frequencies) частоты флуктуаций. При таком делении характерные периоды колебаний изучаемых флуктуаций составят: $T_{\min } \approx 5000 \mathrm{~s}, \quad T_{U L F} \approx 30 \mathrm{~s}, \quad T_{V L F} \approx 13 \mathrm{~s}, \quad T_{L F} \approx 3 \mathrm{~s}$, $T_{H F} \approx 1.3 \mathrm{~s}$. Поведение спектрального интеграла $E_{U L F}(t)$, определяемого формулой (4), для сигнала QFS представлено на рис. 5. Тонкой линией изображены значения $E_{U L F}(t)$, полученные с помощью метода TST на интервалах времени $0<t<t_{\text {off }}$ и $T-t_{\text {off }}<t \leq T$. На интервале времени $t_{o f f}<t<T-t_{o f f}$ значения $E_{U L F}(t)$, полученные без использования метода TST (жирная линия), и с использованием метода TST (тонкая линия), совпадают. Характерная продолжительность вспышки в диапазоне ULF составляет величину $\tau_{U L F} \approx 700 \mathrm{~s}$. Если привести значения $E_{\mu}(t)$ для других спектральных диапазонов $\mu=\{V L F, L F, H F\}$, то эти кривые также показывают сильную нестационарность сигнала QFS. Подтверждением этого факта являются сильно изменяющиеся спектральные интегралы $E_{V L F}(t)$, $E_{L F}(t), E_{H F}(t)$, изображенные на рис. 6-9. Однако характерные величины продолжительностей вспышек

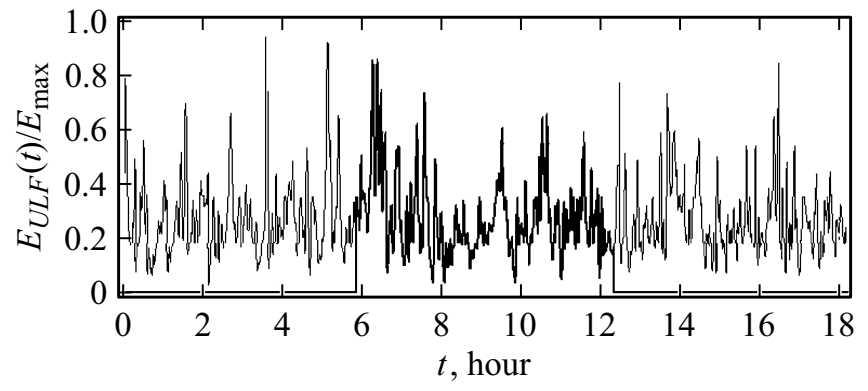

Рис. 5. Зависимость спектрального интеграла $E_{U L F}(t) / E_{\max }$, нормированного на свое максимальное значение, от времени $t$.

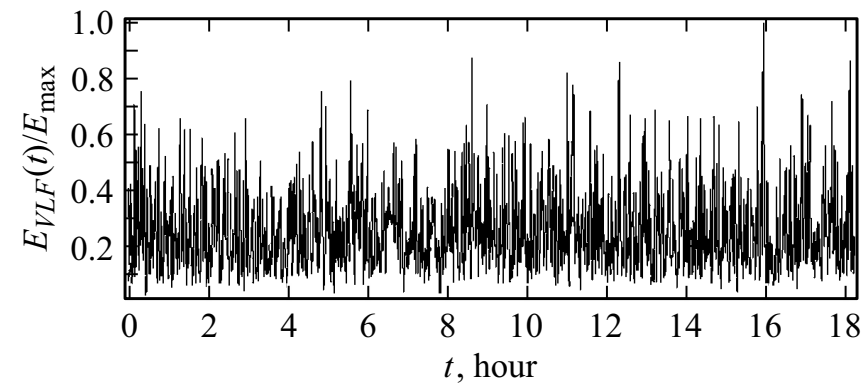

Рис. 6. Зависимость спектрального интеграла $E_{V L F}(t) / E_{\max }$, нормированного на свое максимальное значение, от времени $t$.

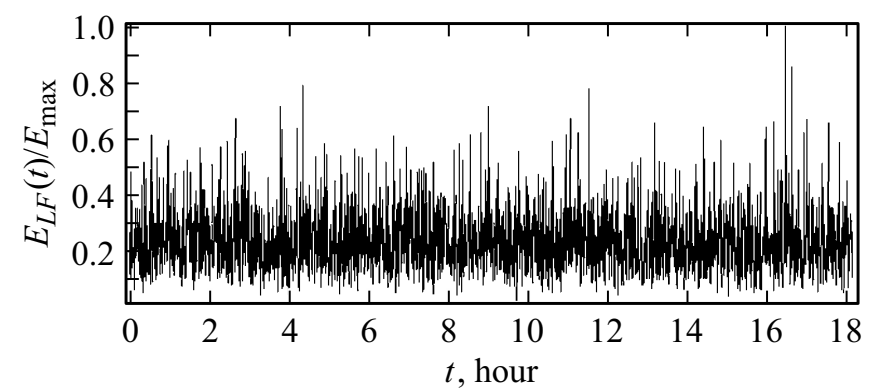

Рис. 7. Зависимость спектрального интеграла $E_{L F}(t) / E_{\max }$, нормированного на свое максимальное значение, от времени $t$.

в таких спектральных интервалах $\tau \approx 130 \mathrm{~s}, \tau_{L F} \approx 40 \mathrm{~s}$, $\tau_{H F} \approx 15 \mathrm{~s}$ становятся меньше.

Таким образом, можно сделать вывод о том, что нестационарный сигнал QFS может быть представлен как совокупность вспышек в различных спектральных диапазонах. Каждая такая вспышка характеризуется временем своего максимума и своей протяженностью во времени.

На рис. 9 вычислено отношение $\frac{\sigma_{W}(n)}{\sigma}$ в зависимости от времени $t_{n}=n \Delta t$. Среднеквадратичное вейвлетотклонение $\sigma_{W}(n)(5)$ найдено с помощью $\mathrm{CWT} V(Q, n)$ сигнала $y(n)$. Заметим, что при численном определении поведения спектральных интегралов $E_{\mu}\left(t_{n}\right)$ и вейвлетдисперсии $\sigma_{W}\left(t_{n}\right)$ необходимо выбирать минимально возможное значение приращения по времени, равное $\Delta t$. Это связано с тем, что все эти вычисляемые величины сильно флуктуируют по времени. При численном расчете $\sigma_{W}(n)$ нижним пределом интегрирования в выражении (5) является величина $Q_{\min }=v_{\min } \Delta t$, а верхним пределом - $Q_{\max }=F_{H F} \Delta t$. Сравнивая рис. 1. с рис. 9, мы видим, что максимальные вспышки сигнала QFS достигают величины $\sqrt{y_{\max }^{2}} / \sigma \approx 4.4$. Для вейвлет-дисперсии $\sigma_{W}^{2}(n)$ максимальная величина $\sigma_{W \max } / \sigma \approx 1.7$. Результат снижения величины $\sqrt{y_{\max }^{2}} / \sigma$ по сравнению с $\sigma_{W \max } / \sigma$ связан с тем, что вейвлет-дисперсия, вычисленная для каждого момента времени $t_{n}=n \Delta t$, представляет собой величину, усредненную как по времени, так по всему диапазону частот. Величина $\sigma_{A l}(\tau)$, основанная на измерении разности средних для двух последовательных из- 


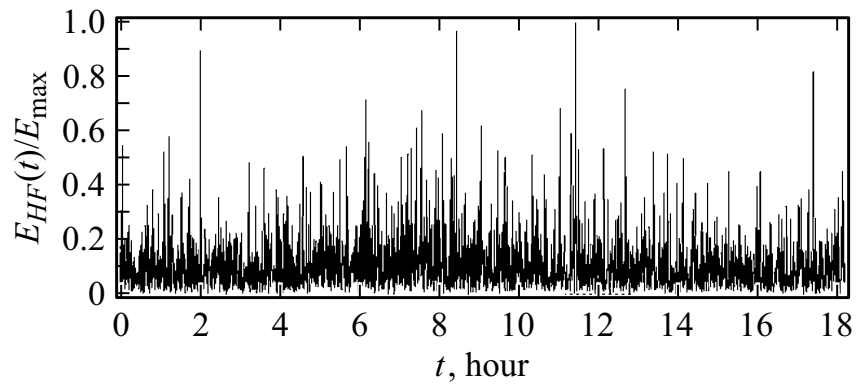

Рис. 8. Зависимость спектрального интеграла $E_{H F}(t) / E_{\max }$, нормированного на свое максимальное значение, от времени $t$.

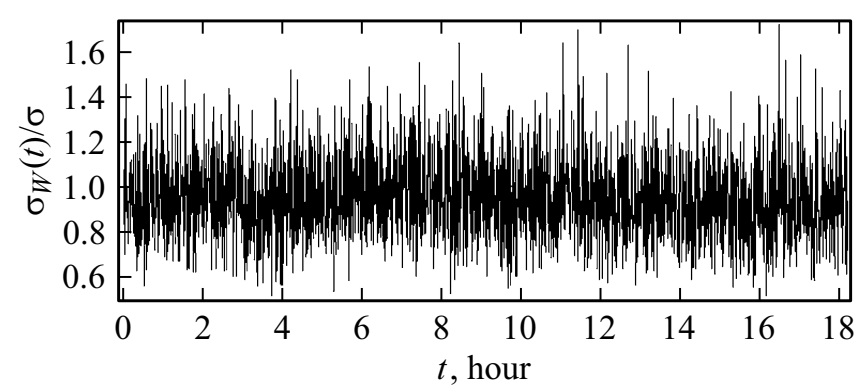

Рис. 9. Зависимость отношения $\sigma_{w}(t) / \sigma$ от времени $t$.

мерений частоты и вычисленная в предположении стационарного эргодического процесса, оказывается примерно на два порядка меньше, чем $\sigma$. Это свидетельствует о том, что введенная нами величина $\sigma_{W}(n)$ соответствует реальным параметрам стабильности QRS.

С математической точки зрения учет граничных условий позволяет выполнить условия теоремы Парсеваля, включив в рассмотрение низкочастотные флуктуации сигнала QRS на границах интервала наблюдения, которые обычно не учитываются. Величина $\sigma_{A l}(\tau)$, основанная на измерении разности средних для двух последовательных измерений частоты и вычисленная в предположении стационарного эргодического процесса, оказывается примерно на два порядка меньше, чем $\sigma$ $\left(\sigma_{A l}(\tau) \ll \sigma\right)$. Подтверждением этого неравенства является анализ слагаемых в формуле суммирования для дисперсии Аллена (1) и сравнение его с равенством Парсеваля. Аргумент времени $\tau$ в формуле Аллена (1) не показывает тех моментов времени, в которых QFS испытывает сильные флуктуации сигнала. Наилучшим показателем развития флуктуаций QFS во времени являются найденные нами спектральные интегралы $E_{\mu}(t)$ в конкретных диапазонах частот $\mu=\{U L F, V L F, L F, H F\}$. Аргумент времени $t_{n}$ для вейвлет-дисперсии $\sigma_{W}^{2}\left(t_{n}\right)$ указывает на моменты развития сильных флуктуаций работы QFS. При вычислении $\sigma_{W}^{2}\left(t_{n}\right)$ производится усреднение флуктуаций по всем спектру частот, а также по интервалу времени, зависящему от этих диапазонов частот. Эти аргументы позволяют сделать вывод о том, что спектральные интегралы и вейвлет-дисперсия позволяют точнее диагностировать стабильность работы QFS.

\section{Заключение}

Проведено обобщение дисперсии Аллена, характеризующей стабильность работы квантового стандарта частоты, на случай нестационарного сигнала. Предложен и развит новый подход, основанный на вейвлетном преобразовании, для анализа нестационарного сигнала квантового стандарта частоты. Непрерывное вейвлетпреобразование сигнала обобщено для дискретного сигнала, показывающего относительное отклонение частоты от ее эталонного значения. Применен метод трансляционного переноса сигнала для устранения граничных эффектов непрерывного вейвлетного преобразования как на начальных, так и на конечных временах изучения сигнала. На основе анализа спектральных интегралов показано, что нестационарный сигнал квантового стандарта частоты представляет собой систему вспышек активности в каждом спектральном диапазоне.

Вычислено значение вейвлет-дисперсии сигнала при усреднении как по времени, так и по всем спектральным диапазонам. Определены моменты времени, в которых наблюдается нестабильность работы квантового стандарта частоты в конкретном спектральном диапазоне.

\section{Финансирование работы}

Работа выполнена при финансовой поддержке Российского фонда фундаментальных исследований № 18-3220022_мол_а_вед.

\section{Конфликт интересов}

Авторы заявляют, что у них нет конфликта интересов.

\section{Список литературы}

[1] J. Vanier. Appl. Phys. B, 81, 421 (2005).

[2] Дж.Л. Холл. УФН, 176 (12), 1353 (2006).

[3] В.Л. Величанский, М.А. Губин. УФН, $179(11), 1219$ (2009). [V.L. Velichansky, M.A. Gubin. Phys. Usp., 52, 1153 (2009)].

[4] Fr. Riehle. Frequency Standards. Basic and Applications (Wiley-VCH, 2004).

[5] А.В. Тайченачев, В.И. Юдин, С.Н. Багаев. УФН, 186, 193 (2016). [A.V. Taichenachev, V.I. Yudin, S.N. Bagayev. Phys. Usp. 59, 184 (2016)].

[6] D.W. Allan. Proc. IEEE, 54 (2), 221 (1966).

[7] А.Г. Чирков, Б.Г. Матисов. Современная теория стабильности прещизионных генераторов (Изд-во Политехнического ун-та, СПб., 2010).

[8] С.А. Зибров, В.Л. Величанский, А.С. Зибров, А.В. Тайченачев, В.И. Юдин. Письма в ЖЭТФ, 82 (8), 534 (2005). [S.A. Zibrov, V.L. Velichansky, A.S. Zibrov, A.V. Taichenachev, V.I. Yudin. JETP Lett., 82 (8), 477 (2005).] 
[9] К.А. Баранцев, Е.Н. Попов, А.Н. Литвинов, В.М. Петров. Радиотехника, 12, 164 (2016).

[10] M.A. Hafiz, G. Coget, M. Petersen, C. Rocher, S. Guérandel, Th. Zanon-Willette, E. de Clercq, R. Boudot. Phys. Rev. Appl., 9, 064002 (2018).

[11] S. Mallat. A Wavelet Tour of Signal Processing, 3rd ed. (Academic Press, NY., 2008).

[12] C.K. Chui. An Introduction to Wavelets (Academic Press, NY., 1992).

[13] I. Daubechies. Ten Lectures on Wavelet (Society for industrial and applied mathematics. Philadelphia, 1992).

[14] O.A. Rosso, M.T. Martin, A. Plastino. Physica A, 347, 444 (2005).

[15] M.E. Pereyra, P.W. Lamberti, O.A. Rosso. Physica A, 379 (1), 122 (2007).

[16] A.E. Hramov, A.A. Koronovckii, V.A. Makarov, A.N. Pavlov. Wavelets in neuroscience (Springer Series in Synergetics, Springer-Verlag, Berlin, Heidelberg, 2015).

[17] С.В. Божокин, С.В. Жарко, Н.В. Ларионов, А.Н. Литвинов, И.М. Соколов. ЖТФ, 87 (6), 822 (2017). [S.V. Bozhokin, S.V. Zharko, N.V. Larionov, A.N. Litvinov, I.M. Sokolov. Techn. Phys., 62 (6), 837 (2017).]

[18] S.V. Bozhokin, N.B. Suvorov. Biomed. Radioelektron, 3, 21 (2008).

[19] S.V. Bozhokin, I.B. Suslova. Physica A, 421, 151 (2015).

[20] С.В. Божокин, К.А. Баранцев, А.Н. Литвинов. Радиотехника, 84 (1(2)), 15 (2020). 